\title{
Las vías abiertas de América Latina
}

\author{
The open roads of Latin America \\ Marcela Fushimi \\ Universidad Nacional de La Plata. Facultad de Humanidades y Ciencias de la Educación. \\ Departamento de Bibliotecologia (UNLP-FaHCE); \\ Instituto de Investigaciones en Humanidades y Ciencias Sociales (IdIHCS-UNLP-Conicet), \\ Argentina \\ mfushimi@fahce.unlp.edu.ar \\ Fernando Ariel López \\ Centro de Innovación de los Trabajadores (CITRA-Conicet); \\ Universidad Metropolitana para la Educación y el Trabajo (UMET); \\ Comunidad Aprender3C, Argentina \\ fernandoariellopez@gmail.com
}

\section{Resumen:}

Se introduce el dossier Del Acceso Abierto a la Ciencia Abierta: avances y reflexiones a 17 años de la BOAI, para lo cual se repasan algunas de las recientes iniciativas surgidas globalmente para avanzar en la concreción del tan ansiado acceso abierto a la literatura científica, con especial énfasis en la región latinoamericana. A la vez se presenta cada uno de los artículos que componen al dossier, el primero de ellos referido al rol de las bibliotecas en los procesos de Acceso abierto y Ciencia Abierta, y los restantes dedicados a análisis situados en tres países de la región: Colombia, Uruguay y Argentina.

Palabras clave: Acceso abierto, Ciencia abierta, América Latina.

\begin{abstract}
:
We present the dossier "On open access to open science: progress and reflections at the age of 17 of the BOAI", in which we review some of the recent initiatives that have emerged around the world to advance the realization of the expected open access to scientific literature, with special emphasis on the Latin American region. At the same time, a summary of the articles included in the dossier is presented, the first referring to the role of libraries in the Open Access and Open Science processes, and the rest dedicated to the analysis of different situations in three Latin American countries: Colombia, Uruguay and Argentina
\end{abstract}

KEYwORDs: Open access, Open science, Latin America.

Cuando nos propusieron coordinar este dossier, había pasado poco más de un año desde el 15 vo. aniversario de la declaración que dio origen al movimiento internacional de acceso abierto -conocida como BOAI o Budapest Open Access Iniciative-, por lo que durante 2017 y 2018 asistimos a eventos conmemorativos, reflexiones y balances que resaltaban, con cierto sabor amargo, los escasos avances alcanzados por el movimiento. Entre ellos, contamos con un extenso texto de Jean-Claude Guédon en el cual realizó un profundo análisis de los diferentes actores involucrados y las dificultades que aún persistían, en sus palabras:

\footnotetext{
Quince años después de la BOAI, sin embargo, la historia nos está enseñando otra vez que tenemos que ser persistentes y pacientes. Mucho ha sucedido, y mucho de eso es positivo, pero hacer un balance de lo que se ha logrado es una tarea urgente, aunque más no sea para tener una idea clara de nuestro rumbo: si bien el acceso abierto ha llegado para quedarse, también despliega una variedad de formatos que no coinciden del todo con el proyecto de inteligencia humana distribuida con el que está asociado. Formas menores y degradadas de acceso abierto han emergido también, algunas veces como resultado de los juegos de poder entre actores poderosos, otras de compromisos propuestos por personas con buenas intenciones. Al mismo
}

Cita sugerida: Fushimi, M. y López, F. A. (2019). Las vías abiertas de América Latina. Palabra Clave (La Plata), 9(1), e076. https:// doi.org/10.24215/18539912e076 
tiempo, la gran multiplicidad de actores sociales involucrados hoy con el acceso abierto ha transformado el campo en algo mucho más complejo de lo que era hace quince años (Guédon, 2017).

En ese texto, Guédon era enfático al señalar que la configuración final que adoptaría el acceso abierto sería el resultado del intercambio de fuerzas que los diversos actores ponen en juego, por lo que no se debería permitir que los intereses económicos interfirieran en el sistema de comunicación científica, que debe ser libre y diseñado para ayudar a la humanidad, "a toda la humanidad, a desarrollar conocimientos". La pregunta que deberíamos hacernos entonces es: ¿quién controla qué?, y si la respuesta nos muestra que no somos nosotros -la comunidad científica- quien lo está haciendo, claramente, estamos en problemas.

Un año más tarde, el conocido grupo canadiense que viene develando con sus incisivas investigaciones el oscuro devenir de la industria editorial (Larivière, Haustein y Mongeon 2015), publicó en 2018 otro lúcido y detallado artículo que nos mostró qué tan lejos nos encontramos aún de alcanzar el objetivo inicial: de un total de 67 millones de artículos científicos publicados con DOI, sólo el $28 \%$ se encontraba en acceso abierto en el momento del estudio, una cifra decepcionantemente baja. Esta investigación también le puso números y colores concretos a las diferentes vías que permiten acceder a ese $28 \%$, evidenciando la diversificación ya anunciada por Guédon el año anterior (Piwowar et al., 2018).

Un poco como respuesta a estos lentos avances, en el ámbito de los países europeos se lanzó en septiembre de 2018 el Plan S, una iniciativa de Science Europe -el consorcio del Consejo Europeo de Investigación y 12 agencias nacionales de financiación de la investigación- que buscó dar un "shock" (por eso la S). El Plan S obligaría a los científicos e investigadores europeos financiados por organizaciones e instituciones estatales a publicar su trabajo en repositorios o revistas abiertas desde 2020. Y para que eso ocurriera, los financiadores se comprometían a cubrir los costos de procesamiento (APC) de los editores comerciales, es decir, solventarían la vía dorada comercial, siempre y cuando se alcance también un acuerdo de costos que sea estandarizado, limitado y justo. La adhesión de los países del "norte global" (Europa + América del Norte) al Plan S fue casi automática, aunque todavía se están realizando ajustes para su implementación. ${ }^{1}$ Sin embargo, en América Latina se levantaron voces de alarma:

\footnotetext{
Desde una perspectiva geopolítica, existen diferencias fundamentales en la noción de publicaciones científicas y académicas, que parecen tratarse como un producto propenso a la comercialización en las pautas del Plan S, mientras que, en América Latina, se conciben como el intercambio comunitario de bienes públicos. La publicación académica en América Latina está respaldada por una infraestructura no comercial y financiada con fondos públicos, orientada a promover el acceso abierto como la forma natural de comunicación científica. En esencia, en nuestra región, los productos científicos pertenecen a la academia y no a las grandes editoriales comerciales internacionales, con una tradición de lectura gratuita y de publicación colaborativa gratuita para acceder al conocimiento, que se considera un derecho universal (Debat y Babini, 2019).
}

Concretamente y como señalan Debat y Babini, el Plan S refuerza y favorece el modelo de publicación comercial, en detrimento de las publicaciones tradicionales latinoamericanas, en su mayor parte editadas por instituciones públicas sin fines de lucro. Y el riesgo que advierten es que con el Plan $\mathrm{S}$ los escasos fondos que disponen estas instituciones vayan a parar a los grandes monopolios editoriales en vez de sostener y ampliar la creciente infraestructura de publicación existente en la región. Infraestructura que se fortaleció a fines de 2018 con la aparición de AmeliCA, una iniciativa impulsada por Redalyc, CLACSO y Unesco, junto a las Universidades de La Plata (UNLP, Argentina), Antioquía (UdeA, Colombia) y Autónoma del Estado de México (UAEM, México). No abundaremos aquí en ella ya que sus bases conceptuales fueron ampliamente descriptas en el dossier del número anterior de Palabra Clave, solo diremos que AmeliCA provee una infraestructura tecnológica y herramientas diversas para facilitar la edición científica de calidad a cargo de las instituciones, y uno de sus lemas es "Conocimiento abierto sin fines de lucro propiedad de la academia”.

Resumiendo lo dicho hasta ahora, tal como en 2002 cuando se realizó la BOAI, las tensiones en el ecosistema de la comunicación científica siguen más vivas y activas que nunca. Por un lado, las editoriales comerciales diversificando sus estrategias e inversiones para continuar maximizando sus ganancias. Y por 
otro, propuestas concretas en América Latina y en Europa que buscan vías sostenibles para la ciencia que sean alternativas posibles al actual modelo. Mientras tanto, los investigadores preocupados por su visibilidad invierten más tiempo en las redes sociales académicas (como ResearchGate, Academia.edu, etc.) que, en autoarchivar su producción en repositorios institucionales o temáticos, fundamentalmente debido a que los mecanismos de autoarchivo son más sencillos y directos que en los repositorios, y les ofrecen una difusión inmediata hacia una red de colegas disciplinar que los conecta (Unzurrunzaga, 2017).

Allá por 2008, Aaron Swartz nos decía en su Manifiesto por la guerrilla del acceso abierto: "No hay justicia alguna en obedecer leyes injustas. Es tiempo de salir a la luz y en la gran tradición de la desobediencia civil, declarar nuestra oposición a este robo privado de la cultura pública”. Sin lugar a dudas, estas palabras calaron fuerte en Alexandra Elbakyan, sobre la situación restrictiva de la comunicación científica por la privatización del conocimiento científico y por los altos costos de compra de los artículos académicos. Esto devino en la fundación de Sci-Hub en 2011, "el primer sitio web pirata del mundo que proporciona acceso público y masivo a decenas de millones de trabajos de investigación" (Sci-Hub). Este proyecto libertario brinda acceso al texto completo de artículos académicos pagos, y aunque su alcance y cobertura no es claro, según un informe (Himmelstein et. al, 2018) "a partir de marzo de 2017, la base de datos de Sci-Hub contiene el 68.9\% de los 81.6 millones de artículos académicos registrados en Crossref y el $85.1 \%$ de los artículos publicados en revistas de acceso gratuito". Por lo que el problema del acceso se atenuó en gran parte gracias a una acción de desobediencia civil, más que por los esfuerzos del movimiento y las políticas públicas implementadas.

En estos 17 años, algunos países de Latinoamérica lograron desarrollar políticas públicas que promueven el acceso abierto al conocimiento científico a través del autoarchivo en repositorios digitales (Perú, Argentina y México), y otros tuvieron o tienen proyectos de ley esperando lograrlo (Brasil, Colombia y Ecuador). En Argentina son conocidos, aunque también lentos los avances logrados a partir de la sanción e implementación de la ley 26.899 en 2013, cuyo portal del Sistema Nacional de Repositorios Digitales cuenta con 35 repositorios e instituciones adheridas de un total de 138 alcanzadas por la ley (Argentina. Ministerio de Educación, Cultura, Ciencia y Tecnología, 2019).

Además a nivel regional, en línea con la vía verde del acceso abierto, no podemos dejar de mencionar a La Referencia, la red latinoamericana de repositorios digitales creada entre 2010 y 2013 que, a partir del trabajo de 10 nodos nacionales (Argentina, Brasil, Chile, Colombia, Costa Rica, Ecuador, El Salvador, México, Perú y Uruguay) y apoyado en la Red Clara, busca brindar visibilidad a la producción científica de las instituciones de educación superior e investigación de América Latina y promueve el acceso abierto y gratuito al texto completo de la producción científica financiada con fondos públicos. La Referencia es un actor clave para la región, ya que forma parte de la Confederación de Repositorios de Acceso Abierto (COAR), colabora en varias líneas de trabajo con el Proyecto OpenAIRE, y un actor de peso tanto para el movimiento de acceso abierto como para las más recientes iniciativas de ciencia abierta en la región.

A nivel internacional, desde 2014, muchos organismos (CEPAL, NSF de EEUU, ICSU, IAP, ISSC, TWAS, Horizon 2020 de la Unión Europea, entre otros) comenzaron a hablar de los beneficios de la gestión de los datos de investigación para promover la innovación económica y social, maximizar las inversiones en Ciencia y Técnica (CyT), asegurar la transparencia, su preservación digital, facilitar el acceso e incentivar su uso y reuso. En nuestra región por el momento hay pocos y esporádicos avances en esta temática y se hace cada día más ineludible trabajar en las instituciones nacionales CyT, centros de investigación y universidades en una hoja de ruta. La misma debería considerar el diseño de políticas de datos de investigación; el desarrollo y mantenimiento de infraestructuras para gestionar planes de gestión de datos de investigación, set de datos, repositorios, etc.; y, la sensibilización y formación de investigadores, estudiantes de posgrados, bibliotecarios y/o gestores institucionales.

Estamos asistiendo a un cambio de paradigma en la forma en que se realiza la ciencia: 
El cambio no está en lo que se hace, sino en cómo se hace. [...] Los dos principales motivos para realizar este cambio son de tipo político-social (la riqueza de la sociedad se debe basar en la innovación) y también científicos. En esta nueva concepción, la ciencia debe ser abierta, colaborativa y hecha con y para la sociedad (Anglada y Abadal, 2018).

En este sentido, en estos últimos años en la región se generaron tres documentos de apoyo y promoción de la ciencia abierta: el Manifiesto de ciencia abierta y colaborativa promovido por la Red de Ciencia Abierta y Colaborativa para el Desarrollo (OCSDNet); la Declaración de Panamá por la ciencia abierta reproducible y replicable en 2018 y el Manifiesto bibliotecario por la ciencia abierta latinoamericana en 2019. En estos manifiestos y declaración bajo con mirada situada se brinda una definición sobre la Ciencia Abierta y los movimientos que lo integran (acceso abierto, datos abiertos, educación abierta, infraestructuras abiertas, hardware abierto, software libre, evaluación abierta y ciencia ciudadana), se argumenta la necesidad de la apertura de las prácticas de investigación y se reconocen a los actores que participan del proceso. Además, se incluyen algunos lineamientos para avanzar hacia una investigación abierta latinoamericana, contextualizada y localizada.

En este marco presentamos el dossier que está compuesto de cuatro artículos que representan miradas diferentes acerca de algunos de los aspectos mencionados en la introducción.

En el primer artículo Jorge Revez -investigador portugués de la Universidad de Lisboa- propone realizar una revisión del rol histórico de las bibliotecas para la colaboración, favorecimiento y fortalecimiento de las prácticas de apertura de la ciencia (durante la producción y su difusión). Realiza, entonces, un recorrido por las tensiones y rupturas de los movimientos que hacen a la Ciencia Abierta. Para esto bucea en un intento por comprender en qué medida la situación actual del acceso abierto, y el paso hacia la ciencia abierta, influyen en el papel de las bibliotecas y su comportamiento frente a estas nuevas dinámicas durante los procesos científicos, sociales y culturales.

El segundo trabajo, pertenece al grupo de investigación de la Universidad de Antioquia en Colombia que es liderado por Alejandro Uribe Tirado, de conocida y prolífica producción académica en temas vinculados al acceso abierto, y forma parte de un conjunto de trabajos realizados en el marco del proyecto "Impacto social, científico y actividades de vinculación con el entorno de centros de investigación de la Universidad de Antioquia". En todos ellos los autores han tomado como objeto de estudio a su propia universidad para analizar el impacto de diferentes aspectos de su actividad, a base de la producción científica publicada con DOI y que está registrada en tres fuentes: Web of Science, Scopus y SciELO. En este trabajo en particular, los autores proponen una interesante metodología para analizar y medir cuánto de su producción científica total está disponible en acceso abierto, por cuáles vías es posible acceder a ella, así como caracterizar su visibilidad e impacto. Es importante resaltar que el estudio reconoce dos importantes limitaciones, a saber: 1) selecciona la producción registrada en las tres fuentes mencionadas (alrededor de 15 mil artículos), dejando fuera del análisis otros tipos de producciones, así como documentos no registrados por esas bases, y 2) considera sólo artículos con DOI, por lo que alrededor de la mitad de los artículos registrados quedó afuera si tenemos en cuenta que su uso se impuso en los inicios del nuevo siglo. No obstante esto, el análisis resulta válido e ilustrativo para caracterizar las tendencias de una parte importante de la producción científica de la UdeA de las últimas décadas. Y su valor adicional reside en que los autores comparten en acceso abierto tanto el algoritmo usado para procesar la información como la base de datos resultante, de modo tal que pueda revisarse y eventualmente reutilizarse para otros estudios. En cuanto a los resultados que obtienen podemos decir que son preocupantes, en tanto muestran que, si bien el porcentaje en abierto es importante y creciente, también han sido crecientes los costos de publicación que la Universidad ha tenido que afrontar para lograrlo. Asimismo, resulta preocupante, y a la vez coincidente con los resultados observados a escala global en estudio mencionado antes (Larivière, Haustein y Mongeon, 2015), el hecho de que más del 60\% de la producción científica de la UdeA se haya publicado en revistas cerradas pertenecientes a tres editoriales oligopólicas: Elsevier, Springer y Wiley. Entre las principales conclusiones del estudio, se destaca la recomendación que los autores hacen en relación con la importancia de reforzar el depósito en el repositorio de la UdeA, como forma 
de garantizar no solo el acceso abierto a su producción sin necesidad de pagar APCs, sino fundamentalmente como el lugar preferencial de registro de la producción y sistema propio de información.

En el tercer artículo, Natalia Aguirre Liguera, Juan Maldini y Exequiel Fontans de la Universidad de La República presentan un recorrido de los avances del acceso abierto en la República Oriental del Uruguay en los últimos diez años. En la primera parte, en base a la producción científica uruguaya registrada por WoS, ${ }^{2}$ encontraron que casi un 38\% de la misma se encuentra disponible en AA real, mientras que otro $50 \%$ podría subirse a los repositorios -AA potencial-. Por otra parte, el relato de los avances en las políticas implementadas a nivel nacional, tanto para fomentar la vía verde como la dorada, demuestran un interés gubernamental sostenido, que recientemente se evidenció con la presentación de SILO, una nueva red de instituciones del sistema científico y tecnológico uruguayo, que promueve el acceso, la preservación y la visibilidad de la producción nacional en ciencia y tecnología a través de repositorios de acceso abierto.

En el último trabajo, las investigadoras argentinas Paola Bongiovani y Sandra Miguel, autoras de buena parte de los estudios argentinos sobre los efectos del acceso abierto tanto en la producción local como en las actitudes, prácticas y opiniones de los científicos en las diferentes disciplinas, presentan en esta oportunidad otro interesante aporte. La pregunta que guía esta nueva indagación y que titula el artículo es: ¿Cuán abierta es la producción científica de los investigadores argentinos de Ciencias Sociales? La respuesta pareciera ser "no tanto", ya que como lo demuestran en su texto, aunque la proporción de artículos en acceso abierto en este segmento disciplinar aumentó en relación con estudios anteriores, también lo han hecho los costos requeridos para lograrlo. El análisis de los montos involucrados por el pago de APCs (tanto de revistas AA como híbridas) y su eventual proyección resulta alarmante, más aún si tenemos en cuenta que hablamos de un área (ciencias sociales) cuya producción total no se difunde mayormente por esta vía, y que claramente tiene costos mucho más bajos que áreas temáticas como las ciencias naturales, exactas, salud, ingeniería y tecnología, etc. Si esto es así para las ciencias sociales, ¿cuánto costaría financiar el pago de APCs para el resto de la producción científica argentina que se publica en el resto de las áreas? Y la pregunta que sigue a continuación sería ¿corresponde al Estado financiar este costo? ¿a quiénes -en realidad- estamos financiando? Consideramos que de las conclusiones del artículo deberían desprenderse líneas y políticas concretas respecto de qué modelo de publicación corresponde apoyar, promover y eventualmente financiar para lograr un acceso abierto real a la producción científica local. En este sentido, sería bueno hacerle llegar los resultados de este estudio a los responsables de la Biblioteca Electrónica de Ciencia y Tecnología de Argentina.

Para finalizar esta introducción, creemos que los artículos incluidos en este dossier constituyen un aporte fundamental para ampliar nuestro conocimiento sobre las características que está tomando el acceso abierto en nuestra región, y de los nuevos roles que las bibliotecas pueden aportar para movilizarlo. En ese sentido, queremos agradecer a los y las autores/as que confiaron en nosotros para difundir sus investigaciones. Y también a los editores de la revista, que nos permitieron coordinar este dossier.

A la vez, esperamos que su lectura anime a reflexionar sobre los desafíos y riesgos actuales, a los efectos de promover en nuestras instituciones políticas de acceso abierto que beneficien y protejan el conocimiento científico como bien común, y su acceso como derecho humano universal. Invitamos a leer y comentar los artículos del dossier.

Mantengámonos persistentes y no tan pacientes, trabajando juntos para alcanzar el acceso y la ciencia abierta en nuestra región.

\section{ReFERENCIAS}

Anglada, L. y Abadal, E. (2018). ¿Qué es la ciencia abierta?. Anuario ThinkEPI, 12, 292-298. https://doi.org/10.31 45/thinkepi.2018.43

Argentina. Ministerio de Educación, Cultura, Ciencia y Tecnología (2019). Políticas institucionales de Acceso Abierto: elaboración y adecuación a la normativa vigente. Presentación realizada en el Segundo Encuentro 
Nacional de Repositorios de Acceso Abierto, 7 de octubre de 2019, Buenos Aires. Recuperado de http://reposito riosdigitales.mincyt.gob.ar/vufind/Content/noticia7

cOAlition S (2019). Plan S: Making full and immediate Open Access a reality. Recuperado de https://www.coalition -s.org/

Debat, H., y Babini, D. (2019, July 11). Plan S en América Latina: Una nota de precaución. [Traducción del original publicado en https://peerj.com/preprints/27834/] http://doi.org/10.5281/zenodo.3332621

Declaración de Panamá por la Ciencia Abierta, Reproducible y Replicable (2018). Recuperado de https://gitlab.com/d eclaraciondepanama/declaracion-de-panama

Guédon, J.-C. (2017). Open Access: Toward the Internet of the Mind. Recuperado de https://www.budapestopenacce ssinitiative.org/boai15/Untitleddocument.docx

Himmelstein, D.S., Rodriguez Romero, A., Levernier, J.G., Munro, T.A., McLaughlin, S.R., Tzovaras, B.G., y Greene, C.S. (2018). Sci-Hub provides access to nearly all scholarly literature. e-Life, 7, e32822. https://doi.org/10.75 54/eLife.32822.001

Larivière, V., Haustein, S., y Mongeon, P. (2015). The Oligopoly of Academic Publishers in the Digital Era. PLOS ONE, 10(6), e0127502. https://doi.org/10.1371/journal.pone.0127502

Manifiesto Bibliotecario por la Ciencia Abierta (2019). Recuperado de https://docutopia.tupale.co/bibliotecariosalse nado-manifiestobibliotecario2019

OCSDNet. (2015) Manifiesto de ciencia abierta y colaborativa: hacia una ciencia abierta e inclusiva por el bienestar social $y$ ambiental. Recuperado de https://ocsdnet.org/wp-content/uploads/2015/04/Manifesto-Infographic-Spanis h-1.pdf

Piwowar, H., Priem, J., Larivière, V., Alperin, J. P., Matthias, L., Norlander, B., ... Haustein, S. (2018). The state of OA: A large-scale analysis of the prevalence and impact of Open Access articles. PeerJ, 6, e4375. https://doi.o $\mathrm{rg} / 10.7717 /$ peerj. 4375

Sci-Hub [sitio web]. Recuperado de: https://www.sci-hub.tw

Swartz, A. (2008). Manifiesto por la Guerrilla del Acceso Abierto. Recuperado de https://es.wikisource.org/wiki/Man ifiesto_por_la_Guerrilla_del_Acceso_Abierto

Unzurrunzaga, C. (2017). Hábitos de los investigadores de Humanidades y Ciencias Sociales para visibilizar sus publicaciones en el entorno digital. Un estudio de caso argentino (Trabajo final de Máster). Universidad de Salamanca. Recuperado de https://gredos.usal.es/jspui/handle/10366/138126

\section{Notas}

1 En base a las críticas recibidas, el deadline del plan S pasó de 2020 a 2021 e incorporó cambios importantes. Entre otros, revalorizó el rol de los repositorios institucionales que en la primera versión del plan estaban contemplados apenas como agentes complementarios y de preservación digital.

2 WoS es la sigla de Web of Science, la base de datos selectiva de Clarivate Analytics que registra las publicaciones de "corriente principal" de la ciencia y con una baja representatividad de revistas latinoamericanas, por lo que una parte importante de la producción no fue analizada. 